\title{
An RF-based Method for Passive Localization of Targets in Wireless Networks
}

\author{
Zhi-Yong Yang, Ming Gao, Lin-Lan Liu \\ School of Software, Nanchang Hangkong University, Nanchang, Jiangxi Province 330063, P.R. China \\ E-mail: yangzhy@ nchu.edu.cn, 15160812Z103@stu.nchu.edu.cn, liulinlan@nchu.edu.cn
}

\begin{abstract}
Device-free means targets not carrying any device. Localization and tracking of device-free targets has attracted much attention due to its potential applications where the targets are not cooperative, such as safe guard system. In this paper, we propose a simple Line-Of-Sight (LOS) model and Hierarchical Clustering Filter and Localization algorithm (HCFLA) to localize and track device-free targets. In wireless sensor networks (WSN), the dynamic values of radio signal strength (RSS) between the static environment and the dynamic environment are measured, which are called RSS values in this paper. From the effected links whose RSS value is more than a threshold, the intersections of these links can be calculated. The proposed HCFLA filter the noise points of the intersections and estimate the number and location of targets use with valuable points. Our experimental test-bed is a 4.5 $\times$ 4.5 square metres area in indoor environment with 18 Micaz sensor nodes evenly distributed on the edge of the square. The experimental results show that the method can detect the number of the targets in high probability, and localize the targets with average error of $0.23 \mathrm{~m}$.
\end{abstract}

Keywords-device-free; localization; clustering; radio frequency; wireless sensor network

\section{INTRODUCTION}

Real-time localization and tracking of moving target is highly in demand and important in many applications, such as patient tracking in hospitals [1], "smart" home and intelligent buildings [2], animal habitat monitoring [3], and battlefield surveillance [4]. It has attracted considerable research interest over the past few years. Global Positioning System (GPS) is a technology well known for its wilder application and accuracy. However, GPS only works in outdoor environments without satellite signals being blocked. Target localization and tracking in indoor environments is more complicated and several technologies, such as video, infrared, and ultrasound, have been used. These technologies are usually costly, including infrastructure, deployment, and maintenance, and may have some restrictions placed on the environments where they are applied. For example, video technology does not work in dark environments and infrared technology does not apply in complicated environments [5].

Radio frequency (RF) is another promising technology, which utilizes radio signal strength (RSS) to localize and track target. The reduction in costs for radio frequency integrated circuits and advances in peer-to-peer data networking have made realistic of RF-based localization. Thus, it has attracted a lot of research interests recently, and many papers have been published in this field [5]-[8]. Localization based on RF technology can be divided into two main categories: device-need localization and device-free localization (DFL) [9]. In the first category, the target has to carry a transmitter, a receiver, or a transceiver. This precondition cannot be met in some application, such as safe guard systems. And DFL is a passive localization approach, which doesn't need the target to carry any device.

In this paper, a novel approach and algorithm based on DFL is proposed to localize target. The basic idea is to collect links whose attenuation is bigger than a threshold in wireless sensor network (WSN), and get the intersections of these links. We propose an algorithm called hierarchical clustering filter and localization algorithm (HCFLA), which can filter out the interference intersections and cluster the residual intersections into several clusters. According to the number of the clusters and their centers, we get the number of targets and their localization.

The rest of this paper is organized as follows. In the next section, we provide some related work. Section 3 introduces the simple line-of-sight (LOS) model and the hierarchical clustering filter and localization algorithm. The experiment and results are showed in section 4. And then we conclude the paper in section 5 .

\section{RELATED WORK}

As mentioned earlier, localization based on RF technology can be divided into device-need localization and device-free localization. Three basic indoor localization models have been studied based on device-need RF technology. They are 802.11, active RFID, and wireless sensor networks.

802.11 technologies use a standard network adapter to measure signal strengths and build a radio map of signal strength value for each location. Signal strength information gathered from known multiple access points can be utilized to localize target which carries a radio detector [6], [10]. Reference [11] utilizes K nearest reference tags' coordinates to calculate the coordinate of the target object who carry an active RFID. It adopts a number of reference tags and RFID readers which are expensive. Similarly, in RF-based WSN, the information including the angle of arrival, distance or he hop number to some nodes whose positions are already known are collected to localize target objects which also carry a sensor node [5], [12].

The other localization based on device-need RF technology is reported in [5], [8], [9]. Three algorithms were proposed to localize and track target in [5]. The midpoint and intersection algorithms are based on points on the influential links. But the two approaches can be used for applications without high accuracy and with only one object. 
The third algorithm is best-cover algorithm, which based on rectangle area created by influential links. The algorithm can be used when there are multiple targets. However, if targets are tightly close to each other, it can't be estimate the number of objects and just recognize them as one target. Reference [8] use radio tomographic imaging (RTI) method to estimate an image of the change in the attenuation as a function of space, and use the image estimate for the purposes of indicating the position of target. But the paper did not address the case of multiple targets are in close proximity from to each other. In this paper, we utilize intersections on the influential links and clustering algorithm to localize multiple targets.

\section{MODEL AND ALGORITHM}

In this section, we describe the method proposed in this paper, including the model and algorithm.

\section{A. Model}

When wireless nodes communicate, the radio signals pass through the physical area of the network. Objects within the area absorb, reflect, diffract, or scatter some of the transmitted power. A wireless signal propagating along a path between a pair of sensors without obstructions (static environment) loses average power with distance is described as [13]:

$$
\bar{P}(d)=P_{t}-P_{0}-10 n_{p} \log _{10}\left(d / d_{0}\right)
$$

where $\bar{P}(d)$ is the average received power at distance $d$ from the transmitting node, $P_{t}$ is the transmitted power, $P_{0}$ is the received power at a reference distance $d_{0}$, and $n_{p}$ is the path loss exponent which controls how fast power is lost along a path. When there is at least one object in the area of wireless sensor network (dynamic environment), the received power on a link between nodes $i$ and $j$ can generally be modeled as

$$
P_{i j}=\bar{P}(d)-X_{i j}-Y_{i j}
$$

where $X_{i j}$ is shadowing loss and $Y_{i j}$ is non-shadowing loss. Thus, the signal attenuation $X_{i j}+Y_{i j}$ on a link allows us to determine whether or not an obstruction lies on its path. In experiment, the RSS can be calculated use Eq. 3 .

$$
R S S=R S S_{\text {static }}-R S S_{\text {dynamic }} .
$$

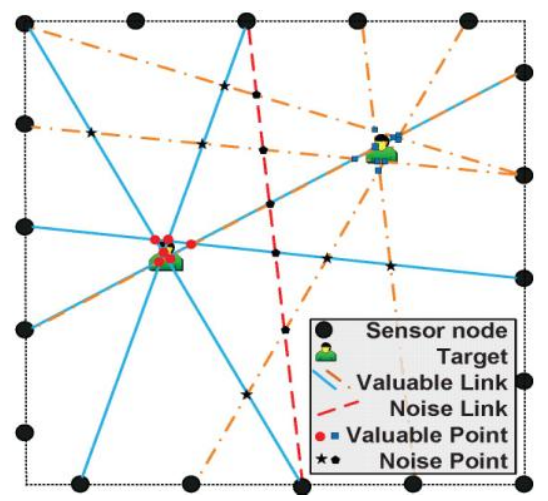

Figure 1. Illustration of the model and algorithm.

where $R S S_{\text {static }}$ is links' RSS when the environment is empty, and $R S S_{\text {dynamic }}$ is links' RSS when there is at least one object in the experiment environment.

Now we consider a wireless network, in which every node communicates with each other. If $\mathrm{K}$ is the number of node, then the total number of unique two-way links is $\mathrm{M}=$ $\mathrm{K}(\mathrm{K}-1) / 2$. Any pair of nodes is counted as a link. The difference of radio power attenuation between dynamic and empty of a link can be calculated using Eq. 3. Generally, the power attenuation difference of the link with an object on its line-of-sight (LOS) path is greater than the link with the object on its non-line-of sight(NLOS). If a link's attenuation difference is much than a threshold $\lambda$, we take the link as effected link, and other links as free links. For example, Fig. 1 is an illustration of LOS and NLOS path of an object. We can see the effected links cross with each other around the object. But there are also some intersections which are far away from the targets, called noise points in this paper. We should throw them away in the first place, then utileze the left intersections as valuable points, which are used by the proposed HCFLA algorithm to localize the targets.

\section{B. Algorithm}

As we have mentioned above, we should filter out the noise points and use the valuable points to localize the targets, which is done by the proposed algorithm called Hierarchical Clustering Filter and Localization Algorithm. The algorithm filter out the noise points firstly, and then cluster the valuable points into different clusters to localize targets using the centers of the clusters. HCFLA can be described in detail as follows: We mark the intersections matrix as A, whose rows correspond to intersections, and columns correspond to intersection's coordinates, including the valuable points and noise points. We use $\mathrm{m}$ correspond to the sum of number of intersections in all clusters, $n$ correspond to the number of clusters, and $n_{C_{i}}$ correspond to the number of intersections in the cluster $C_{i}$, Subscript $i$ refers to the sequence number of the cluster.

Step 1: Each intersection in $A$ is initialized as a cluster, and the cluster center is itself. 
Step 2: Calculate the Euclidean distance of all clusters' centers.

Step 3: For each cluster $C_{i}(i \in[1, n])$, If the nearest distance from $C_{i}$ to other clusters is bigger than the threshold noise_d and if $m / n_{C_{i}}>\gamma$ ( $\gamma$ is a threshold), delete the cluster $C_{i}$ as noise points, set $n=n-1$ and $m=m-n_{C_{i}}$.

Step 4: Find the smallest distance between cluster $C_{i}$ and $C_{j}(i \in[1, n-1]$ and $j \in[2, n])$, combine $C_{i}$ and $C_{j}$ into $C_{i}$, then calculate the center of the new cluster $C_{i}$ and delete $C_{j}$, set $n=n-1$.

Step 5: If $n>3$, go to Step 2. Else go to Step 3.

Step 6: For each pair of clusters, if their distance is smaller than the threshold target_d, combine the two clusters and set $n=n-1$.

Step 7: Return $n$, the valuable points and the center of $C_{i}(i \in[1, n])$.

\section{EXPERIMENTAL RESULTS}

In this section, we first describe our experimental setup. Second, the parameters of the proposed algorithm are given. Third, we show the experimental results with one, two and three people in the wireless network area.

\section{A. Physical Description Of Experiment}

A wireless network containing 18 Micaz nodes was deployed in a laboratory. Each node is placed 1.0 miter apart along the perimeter of a $4.5 \times 4.5$ square metres and 1.0 miter off the ground on a tripod. Each node operates in the 2.4G frequency band, and run the IEEE 802.15.4 standard protocol for communication. The transmission power of the nodes were set at $-10 \mathrm{dBm}$. A base station listens to all network traffic and feeds the data to a laptop computer via a COM port. In this experiment, the system is calibrated by taking RSS measurements while the network is vacant from objects. The data collection period of vacant situation is over 50 seconds, approximate 1100 samples from each link. The period of situation with objects is much shorter, about 2 seconds. The final each link's RSS is an average of all samples.

\section{B. Setting Of Parameters}

The threshold $\lambda$ is a key point for deciding the effected links. If its value is set very high, there are no enough effected links to localize the targets. On the contrary, many effected links caused by noise which we called as noise link will be taken into account, which will decrease the accuracy making a tradeoff necessary. Another two important thresholds are noise ${ }^{d} d$ and $\gamma$, which are key points for deciding an intersection is valuable point or noise point. If the value of noise_d is set very high and the value of $\gamma$ is set very low, some intersections not around the targets will be taken as valuable points, on the contrary, some intersections around the targets will be taken as noise point, which both will decrease the localization accuracy. The Parameter target_ $d$ is determined by the resolution of the system. In this paper, we set ${ }^{\text {target }}{ }^{d}$ as 0.5 metre, which is the expecting resolution of our system. All the parameters are set as in Table I.

TABLE I. ALGORITHM PARAMETERS SETTING

\begin{tabular}{|c|c|c|}
\hline Parameter & Value & Description \\
\hline$\lambda$ & 4 & $\begin{array}{c}\text { Threshold for choose effected } \\
\text { links (dB) }\end{array}$ \\
\hline noise_d & 0.3 & $\begin{array}{c}\text { Threshold for filter out valueless } \\
\text { point (m) }\end{array}$ \\
\hline$\gamma$ & 5 & $\begin{array}{c}\text { Threshold for filter out valueless } \\
\text { point }\end{array}$ \\
\hline target_d & 0.5 & $\begin{array}{c}\text { Threshold for distinguish } \\
\text { different targets (m) }\end{array}$ \\
\hline
\end{tabular}

\section{Localization Of Targets}

As the floor of the laboratory is proved with tiles with size of $0.5 \times 0.5 \mathrm{~m}$, we number the tiles in the wireless network area from 1 to 81 and use them to mark location. In our experiments, targets always stood at centers of different tiles, so the least distance between two targets is $0.5 \mathrm{~m}$. Depending on the different number of target, we conducted four groups of experiments, each group include fifteen experiments. In the first group, there is only one target standing at the experimental area. We conducted fifteen experiments with the target stood at center of different tile, which were got randomly by Matlab. All the fifteen experiments are detected as there is one target correctly by our algorithm, as list in Table II, and the localization error is under $0.3 \mathrm{~m}$. We give an example of localization result with one target stood at coordinate $(1.25,0.75)$ in Fig.2.

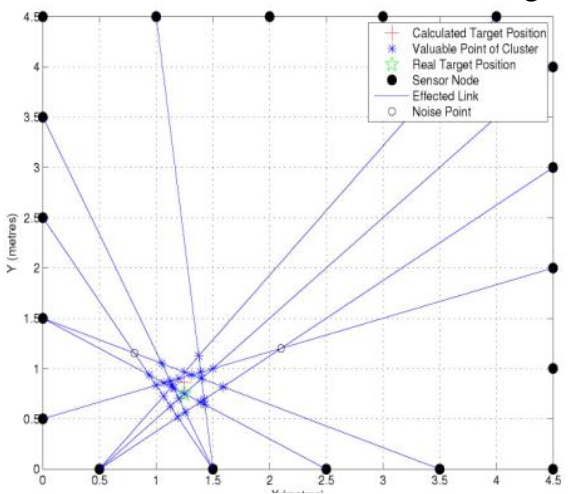

Figure 2. The HCFLA result with one target standing at coordinate $(1.25,0.75)$ 
TABLE II.

RESULTS OF TARGETS LOCALIZATION

\begin{tabular}{|c|c|c|c|c|}
\hline Experiment & $\begin{array}{c}\text { Number of } \\
\text { Experiment }\end{array}$ & $\begin{array}{c}\text { Number of correct } \\
\text { Experiment }\end{array}$ & Correct Rate & Average Error \\
\hline One Target & 15 & 15 & $100 \%$ & 0.14 \\
\hline Two Targets & 15 & 15 & $73 \%$ & 0.41 \\
\hline Three Targets & 15 & 11 & $90 \%$ & 0.23 \\
\hline All Experiments & 60 & 54 & & \\
\hline
\end{tabular}

In the second groups, there are two targets. The locations of the two targets were got totally randomly in the same way introduced above. Fig. $3 \mathrm{a}$ is an example of localization result with two targets stood at coordinates $(1.25,1.75)$ and $(2.25,3.75)$. In Table II we can see that all fifteen experiments are detected as there are two targets correctly. And only one of the localization errors is about $0.5 \mathrm{~m}$, the others are below $0.3 \mathrm{~m}$.

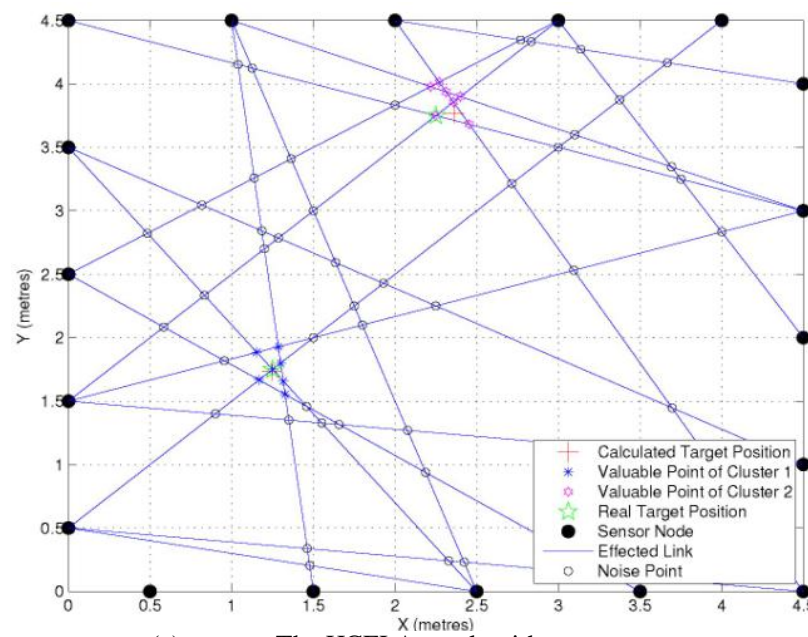

(a)

The HCFLA result with two targets

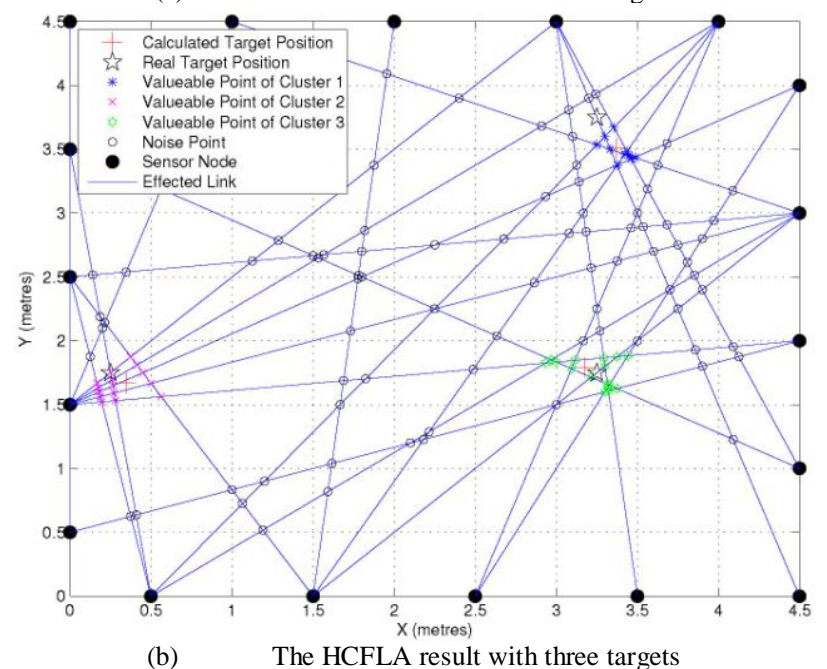

Figure 3. Experimental results of multiple targets.
In the third group, there are three targets. The three locations were also got totally randomly as above. The effected links and intersections increase as the targets. So it is much difficult to detect valuable point and noise point. In fifteen experiments, there are eleven are detected correctly as there are three targets, as showed in Table II. And the localization error is much bigger than one or two targets. The biggest localization error is about $1 \mathrm{~m}$. An illustration with three targets stood at coordinates $(0.25,1.75)$, $(3.25,1.75)$ and $(3.25,3.75)$ is showed in Fig. 3b.

\section{$\mathrm{V}$ CONCLUSION}

This paper proposed an algorithm for localizing device-free objects in an indoor environment using RF-based technology. The method utilizes the RSS dynamic value between the static environment and the dynamic environment in WSN to calculate the position of target. The proposed HCFLA algorithm gets the intersections of effected links whose attenuation is more than a threshold, then filters the noise points of the intersections and use the valuable points to estimate the number and locations of targets.

Experiments were conducted in a $4.5 \times 4.5 \mathrm{~m}$ area in indoor environment with $18 \mathrm{Micaz}$ sensor nodes, which were evenly distributed on the edge of the square on tripods. The experimental results show that the method can detect the number of targets when there are no more than three targets in the WSN area in high probability. And the total average localization error is approximate $0.23 \mathrm{~m}$. Our future direction in this area involves trying a larger area covered by sensor nodes, localizing of more than three targets, and tracking multiple moving targets.

\section{ACKNOWLEDGEMENT}

This work is supported in part by grants from the National Natural Science Foundation of China (nos. 61501218, 61363015, and 61501217).

\section{REFERENCES}

[1] T. Gao, D. Greenspan, M. Welsh, R. Juang, and A. Alm, "Vital signs monitoring and patient tracking over a wireless network," in Proc. 27th Annual Int. Conf. of the Engineering in Medicine and Biology Society IEEE-EMBS (2005) 102-105.

[2] Estrin, R. Govindan, and J. Heidemann, "Embedding the internet: introduction," Commun. ACM, 43(2000) 38-41. 
[3] Tovar, T. Friesen, K. Ferens, and B. McLeod, A DTN wireless sensor network for wildlife habitat monitoring. In: 23rd Canadian Conf. on Electrical and Computer Engineering (CCECE), (2010) 1-5.

[4] R. Bansal, Microwave surfing - which came first: big bang or big crunch?. IEEE Microwave Magazine, 2(2) (2001) 32-34.

[5] Zhang, J. Ma, Q. Chen, and L. M. Ni, An rf-based system for tracking transceiver-free objects. In: 5th Annual IEEE International Conference on Pervasive Computing and Communications, White Plains, NY, (2007) 135-144.

[6] P. Bahl and V. N. Padmanabhan, "Radar: an in-building rf-based user location and tracking system," in Proc. IEEE Nineteenth Annual Joint Conf. of the IEEE Computer and Communications Societies INFOCOM 2 (2000) 775-784.

[7] Viani, L. Lizzi, P. Rocca, M. Benedetti, M. Donelli, and A. Massa, "Object tracking through rssi measurements in wireless sensor networks," Electronics Letters, 44(10)(2008) 653-654.

[8] J. Wilson and N. Patwari, "Radio tomographic imaging with wireless networks," IEEE Transactions on Mobile Computing, 9(5) (2010) 85-94.
[9] M. Youssef, M. Mah, and A. Agrawala, "Challenges: device-free passive localization for wireless environments," in Proceedings of the 13th annual ACM international conference on Mobile computing and networking, MobiCom '07. New York, NY, USA: ACM, (2007) 222-229.

[10] J. Yin, Q. Yang, and L. Ni, "Adaptive temporal radio maps for indoor location estimation," in Proc. Third IEEE Int. Conf. Pervasive Computing and Communications, (2005) 85-94.

[11] L. M. Ni, Y. Liu, Y. C. Lau, and A. P. Patil, "Landmarc: indoor location sensing using active rfid," in Proc. First IEEE Int. Conf. Pervasive Computing and Communications (PerCom 2003), (2003) 407-415.

[12] Niculescu and B. Nath, "Ad hoc positioning system (aps) using aoa," in Proc. INFOCOM 2003. Twenty-Second Annual Joint Conf. of the IEEE Computer and Communications. IEEE Societies, 3(2003) 1734-1743.

[13] N. Patwari and P. Agrawal, "Effects of correlated shadowing Connectivity, localization, and rf tomography," in Proc. Int. Conf. Information Processing in Sensor Networks, (2008) 82-93. 